Article

\title{
Application of High-Performance Liquid Chromatography Coupled with Linear Ion Trap Quadrupole Orbitrap Mass Spectrometry for Qualitative and Quantitative Assessment of Shejin-Liyan Granule Supplements
}

\author{
Jifeng Gu ${ }^{1,2}$, Weijun Wu ${ }^{1}$, Mengwei Huang ${ }^{1}$, Fen Long ${ }^{1}$, Xinhua Liu ${ }^{1}$ and Yizhun Zhu ${ }^{1,3, *}$ \\ 1 Shanghai Key Laboratory of Bioactive Small Molecules, Department of Pharmacology, School of Pharmacy, \\ Fudan University, Shanghai 201203, China; jifeng.gu@fdeent.org (J.G.); 13211030034@fudan.edu.cn (W.W.); \\ 16211030050@fudan.edu.cn (M.H.); 13301030080@fudan.edu.cn (F.L.); liuxinhua@fudan.edu.cn (X.L.) \\ 2 Department of pharmacy, Eye \& ENT Hospital of Fudan University, Shanghai 200031, China \\ 3 State Key Laboratory of Quality Research in Chinese Medicine and School of Pharmacy, Macau University of \\ Science and Technology, Macau, China \\ * Correspondence: yzzhu@must.edu.mo; Tel.: +853-8879-2880
}

Received: 11 March 2018; Accepted: 7 April 2018; Published: 11 April 2018

\begin{abstract}
A method for high-performance liquid chromatography coupled with linear ion trap quadrupole Orbitrap high-resolution mass spectrometry (HPLC-LTQ-Orbitrap MS) was developed and validated for the qualitative and quantitative assessment of Shejin-liyan Granule. According to the fragmentation mechanism and high-resolution MS data, 54 compounds, including fourteen isoflavones, eleven ligands, eight flavonoids, six physalins, six organic acids, four triterpenoid saponins, two xanthones, two alkaloids, and one licorice coumarin, were identified or tentatively characterized. In addition, ten of the representative compounds (matrine, galuteolin, tectoridin, iridin, arctiin, tectorigenin, glycyrrhizic acid, irigenin, arctigenin, and irisflorentin) were quantified using the validated HPLC-LTQ-Orbitrap MS method. The method validation showed a good linearity with coefficients of determination $\left(\mathrm{r}^{2}\right)$ above 0.9914 for all analytes. The accuracy of the intra- and inter-day variation of the investigated compounds was $95.0-105.0 \%$, and the precision values were less than $4.89 \%$. The mean recoveries and reproducibilities of each analyte were $95.1-104.8 \%$, with relative standard deviations below $4.91 \%$. The method successfully quantified the ten compounds in Shejin-liyan Granule, and the results show that the method is accurate, sensitive, and reliable.
\end{abstract}

Keywords: Shejin-liyan Granule; HPLC-LTQ-Orbitrap MS; identification; quality assessment

\section{Introduction}

Pharyngitis is an inflammation of the pharyngeal mucous membrane and submucous lymphoid tissues that affects people of all ages around the world. Patients often report pain and irritation in the throat. In certain cases, the illness is not caused by an infection; in such a setting, antibiotics may be the wrong choice for treatment or they may only offer a modest improvement in symptoms $[1,2]$. Traditional Chinese medicines have been successfully used to treat pharyngitis for thousands of years because of their variety of multi-target, therapeutic, and synergistic effects $[1,3,4]$.

Shejin-liyan Granule (SJLYKL) is a traditional Chinese herbal medicine that has been used to treat pharyngitis in clinical practice. It consists of six herbs: Rhizoma Belamcandae, Arctium lappa, radix Sophorae tonkinensis, Physalis alkekengi, radix Platycodonis, and radix Glycyrrhizae. These chemicals, such as the isoflavones from Rhizoma Belamcandae [5], physalins from Physalis alkekengi [6], ligands 
from Arctium lappa [7], and alkaloids from Sophorae tonkinensis [8] have been proven to have good anti-inflammatory, antibacterial, and antioxidant activities [9-11]. SJLYKL is an organic combination of complex and diverse chemical constituents from these six herbs, and its anti-pharyngitis effect may be closely related to these compounds. Therefore, systematically analyzing the constituents of SJLYKL could provide an interpretation of the material basis for its pharmacological effects.

The complete profiles and quantities of the bioactive ingredients in SJLYKL are not well understood. Several studies have focused on identifying the components in each of the herbs included in SJLYKL [5,6,12-14]; however, no method has been developed to systematically analyze SJLYKL. Therefore, it is important to develop a systematic qualitative and quantitative evaluation method for the pharmacologically active compounds in SJLYKL.

Liquid chromatography-mass spectrometry (MS) has become increasingly common because of its high selectivity and sensitivity. The combination of a linear ion tap with a high-resolution Orbitrap analyzer has been used for qualitative and quantitative analyses in various applications, including bioactive compounds of traditional Chinese medicines, metabolites, and drug abuse [15-17]. In this study, we developed a simple and rapid method for the comprehensive qualitative and quantitative analyses of the major constituents of SJLYKL. This is the first time that a technique using high-performance liquid chromatography coupled with linear ion trap quadrupole Orbitrap high-resolution mass spectrometry (HPLC-LTQ-Orbitrap MS) has been applied to identify and quantify the components in SJLYKL.

\section{Materials and Methods}

\subsection{Reagents and Materials}

Chlorogenic acid, neochlorogenic acid, cryptochlorogenic acid, arctiin, arctigenin, tectoridin, tectorigenin, iridin, irisflorentin, irigenin, matrine, oxymatrine, liquiritin, isoliquiritin, liquiritigenin, isoliquiritigenin, and galuteolin were obtained from the Shanghai YuanYe Biotechnology Co., Ltd. (Shanghai, China) for use as references. Glycyrrhizic acid and glycyrrhetinic acid were purchased from China's National Institute for the Control of Pharmaceutical and Biological Products (Beijing, China). The purity of the reference compounds was $>98 \%$ based on high-performance liquid chromatography (HPLC) analysis. SJLYKL was prepared by the Shanghai Liantang pharmacy (Shanghai, China). Deionized water was purified using a Milli-Q system (Millipore, Bedford, MA, USA). Ammonium acetate (HPLC grade; purity $\geq 98.0 \%$ ) and acetic acid (HPLC grade; purity $\geq 99.7 \%$ ) were provided by ANPEL Laboratory Technologies (Shanghai, China). Chromatographic grade methanol and acetonitrile were purchased from Merck (Darmstadt, Germany).

\subsection{Sample Preparation}

SJLYKL (0.1 g) was accurately weighed and extracted with $10 \mathrm{~mL}$ of $75 \%$ methanol in an ultrasonic bath at room temperature. The weight loss was compensated by adding $75 \%$ methanol after extraction, and the extract was centrifuged at $17,000 \times g$ for $10 \mathrm{~min}$. The resulting supernatant was diluted by a factor of 10 using pure water; then, a $10-\mu \mathrm{L}$ aliquot of the supernatant was injected into the HPLC-LTQ-Orbitrap MS system for analysis. Samples that were above the upper limit of quantification were diluted and reanalyzed.

\subsection{Chromatographic and Mass Conditions}

Chromatographic separation was performed on a Dikma ODS C 18 column $(150 \times 4.6 \mathrm{~mm}, 5 \mu \mathrm{m})$ maintained at $30^{\circ} \mathrm{C}$ using a Dionex HPLC system (Thermo Fisher Scientific, Sunnyvale, CA, USA). The gradient elution was $30 \mathrm{~min}$ at a flow rate of $1.0 \mathrm{~mL} / \mathrm{min}$ using solvent A (water with $0.1 \%$ acetic acid and $2 \mathrm{mM}$ ammonium acetate in water) and solvent B (acetonitrile) as the mobile phase. The elution was run on the following schedule: $10-20 \% \mathrm{~B}$ at $0-5 \mathrm{~min}, 20-50 \% \mathrm{~B}$ at $5-20 \mathrm{~min}, 50-60 \%$ B 
at $20-21 \mathrm{~min}, 60-90 \% \mathrm{~B}$ at $21-25 \mathrm{~min}, 90 \% \mathrm{~B}$ at $25.1-30 \mathrm{~min}, 90-10 \% \mathrm{~B}$ at $30-30.1 \mathrm{~min}$, and $10 \% \mathrm{~B}$ at 30.1-36 min.

For qualitative analysis, the Orbitrap resolution of the survey scan was set to 30,000 and that for the MSn scan was set to 15,000. The data-dependent MS2 scanning was performed to trigger fragmentation spectra of the target ions and to prevent repetition by the dynamic exclusion settings. Peaks were identified by comparison with those of the standards. For those peaks that did not correspond to the standards, a database including about 200 major compounds was established by collecting information from the literature on the six herbs in SJLYKL, including their names, formulas, accurate molecular weights, and MS2 information. The accurate masses of the additive ions, such as $[\mathrm{M}+\mathrm{H}]^{+},[\mathrm{M}+\mathrm{Na}]^{+}$, $[\mathrm{M}-\mathrm{H}]^{-}$, and $\left[\mathrm{M}+\mathrm{HCOO}^{-}\right.$, were also calculated. The MS detection was performed in selected ion monitoring mode to quantify the ten compounds, including matrine, galuteolin, tectoridin, iridin, arctiin, tectorigenin, glycyrrhizic acid, irigenin, arctigenin, and irisflorentin.

\subsection{Method Validation for Quantitative Analysis}

Stock standard solutions of these ten compounds were separately prepared in methanol and kept at $4{ }^{\circ} \mathrm{C}$. A mixed working solution was prepared before each use and diluted to the appropriate concentration to create calibration curves. The calibration curve of each compound was prepared using at least five different concentrations. The external standard method was constructed using the area with respect to known concentrations of the test compound $(C, \mu \mathrm{g} / \mathrm{mL})$ and weighting of reciprocal concentrations $\left(1 / C^{2}\right)$. The limits of detection (LOD) under the present chromatographic conditions were determined at a signal-to-noise ratio of 3.

The intra-day and inter-day accuracies (deviation from the nominal concentration (\%)) and precisions (relative standard deviation, RSD\%) were analyzed at different concentrations on one day, and this experiment was repeated for three consecutive days.

To evaluate the recovery of the method, known amounts of these compounds were added to SJLYKL, and the samples were then quantified as described above. The recovery of each analyte was calculated according to the following equation:

$$
\text { Recovery }(\%)=\frac{A_{\text {det }}-A_{\text {orig }}}{A_{\text {spi }}} \times 100
$$

where $A_{\text {det }}$ is the total detected amount of each compound, $A_{\text {orig }}$ is the original amount of each compound in SJLYKL, and $A_{\text {spi }}$ is the spiked known amount of each component. The amount of the target compound was calculated using the corresponding calibration curve.

To investigate the repeatability, five samples from the same batch of SJLYKL were accurately weighed and treated as described above. The sample stability was assessed by analyzing SJLYKL samples stored at $4^{\circ} \mathrm{C}$, after $0,4,8$, and $24 \mathrm{~h}$.

\section{Results and Discussions}

\subsection{Development of the Extraction Method}

The factors that affect the extraction efficiency, including the extraction solvents, extraction method, and extraction times, were investigated to optimize the sample extraction efficiency. The ultrasonic bath extraction method was convenient and effective for the examined components; therefore, further experiments were performed using ultrasonic bath extraction. Several components, such as arctiin, arctigenin, tectoridin, and tectorigenin, were not completely extracted using pure methanol as a solvent; thus, different water-methanol ratios (0:100, 25:75, and 50:50, v/v) were screened. The yield of arctiin, arctigenin, tectoridin, and tectorigenin increased significantly when extractions were performed with $75 \%$ or $50 \%$ methanol, and fewer interfering peaks were found when $75 \%$ methanol was used. The duration of the extraction (30 min, $40 \mathrm{~min}, 50 \mathrm{~min}$, or $60 \mathrm{~min}$ ) was also 
investigated to optimize the extraction procedure. The optimal extraction of SJLYKL (0.1 $\mathrm{g}$ of powder) was obtained using $10 \mathrm{~mL}$ of $75 \%$ methanol in an ultrasonic water bath for $30 \mathrm{~min}$.

\subsection{Profiles of Ingredients in the SJLYKL Extract}

In the analysis of the obtained chromatographic peaks by the HPLC-LTQ-Orbitrap MS method, we excluded the peaks which were the obtained parent accurate molecular weights without product ion. Then, to prevent misunderstanding caused by interference peaks, some peaks with an absolute intensity lower than 104 were removed. As a result, by comparing the information collected from the literature and standards with the data obtained by the HPLC-LTQ-Orbitrap MS method mass spectrometry, a total of 54 compounds were identified from the SJLYKL extract, including fourteen isoflavones, eleven ligands, eight flavonoids, six physalins, four triterpenoid saponins, six organic acids, two xanthones, two alkaloids, and one licorice coumarin (Table 1). The chemical structures of the 54 compounds are available online as Supplementary Materials. Typical peak chromatograms in positive and negative ion modes are shown in Figure 1.

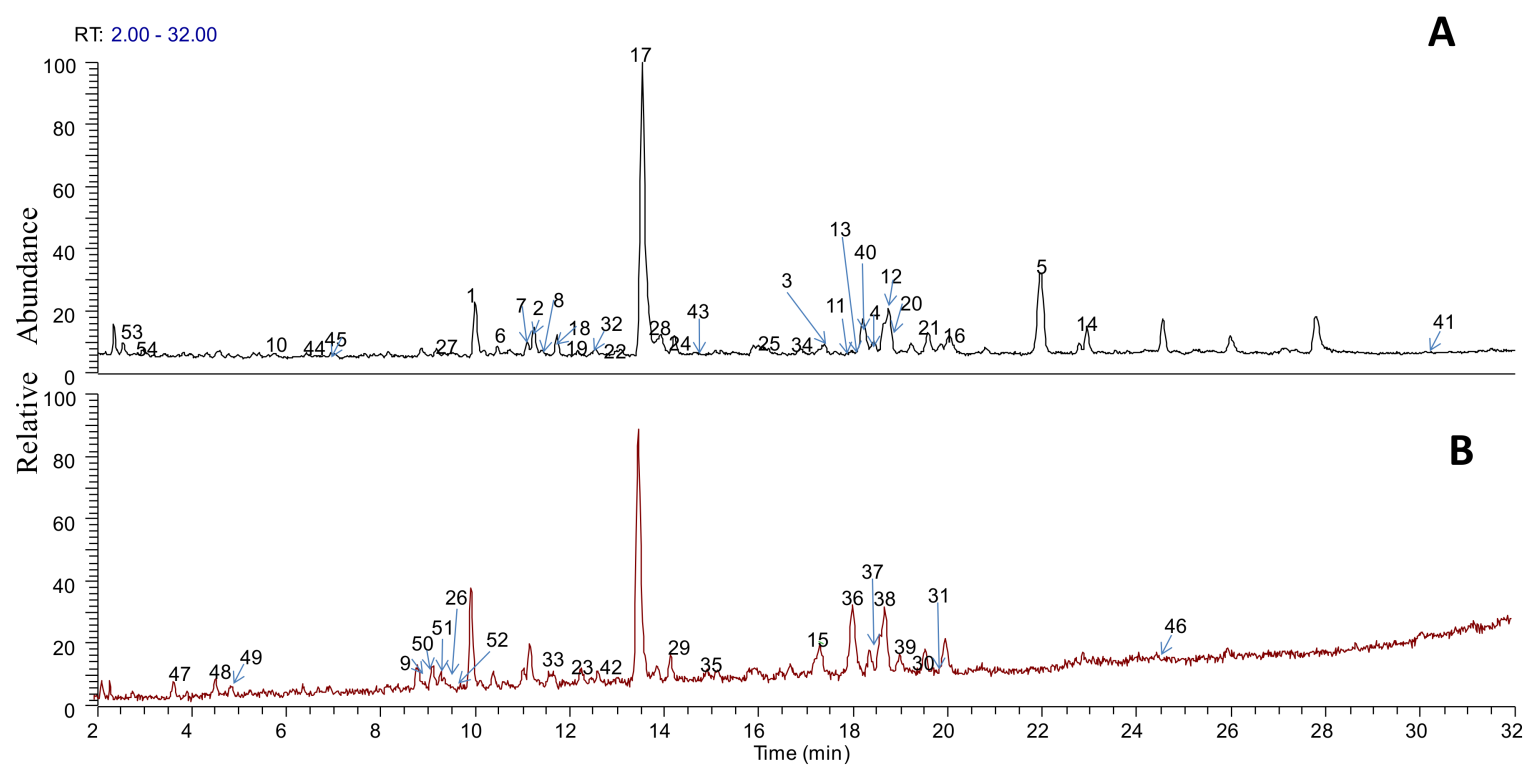

Figure 1. Base peak chromatogram of extract of Shejin-liyan Granule in positive mode (A) and negative mode (B).

Fourteen isoflavones, including seven isoflavone O-glucosides, four aglycones, and three isoflavones with methylenedioxy groups, were unambiguously or tentatively identified [18]. By comparing the retention times and the MS spectra of the SJLYKL extract with those of the standards, five peaks were unambiguously characterized as tectoridin (peak 1), iridin (peak 2), tectorigenin (peak 3), irigenin (peak 4), and irisflorentin (peak 5). Peaks 6, 7, 8, and 9 exhibited a high intensity $[\mathrm{M}+\mathrm{H}-162]^{+}$ion, and were tentatively characterized as iristectorin B, iristectorin A, isoiridin, and 3,5-dimethoxyirisolone-4-O-glucoside, respectively, based on daughter ions [19]. Peak 10 showed a loss of $162 \mathrm{Da}$ at $m / z 463.1236$ and an aglycon ion [M $+\mathrm{H}-162-162]$ at $\mathrm{m} / \mathrm{z} 301.0707$; therefore, this compound was inferred as tectorigenin-7-O-glucosyl-4-O-glucoside [5]. Besides known aglycones, peaks 11 and 12 shared the same molecular ions and fragment pathways, and they were deduced as iristectorigenin A and iristectorigenin B, respectively, based on reported data [5]. Peaks 13 and 14 yielded diagnostic ions at $\left[\mathrm{M}+\mathrm{H}-\mathrm{CH}_{3}\right]^{+}$and $\left[\mathrm{M}+\mathrm{H}-2 \mathrm{CH}_{3}-\mathrm{CO}\right]^{+}$, so they were tentatively characterized as noririsflorentin and dichotomitin, respectively [13]. 
Table 1. Identified compounds of Shejin-liyan Granule by HPLC-LTQ-Orbitrap method.

\begin{tabular}{|c|c|c|c|c|c|c|}
\hline No. & Retention Time (min) & Experimental Mass $(\mathrm{m} / \mathrm{z})$ & Theoretical Mass $(\mathrm{m} / \mathrm{z})$ & Ion Type & Identification Compound & MS/MS Fragments \\
\hline 1 & 9.99 & 463.1237 & 463.124 & {$[\mathrm{M}+\mathrm{H}]^{+}$} & tectoridin & $301.0706,286.0472$ \\
\hline 2 & 11.23 & 523.1447 & 523.1342 & {$[\mathrm{M}+\mathrm{H}]^{+}$} & iridin & 361.0919 \\
\hline 3 & 17.38 & 301.0706 & 301.0711 & {$[\mathrm{M}+\mathrm{H}]^{+}$} & tectorigenin & 286.0475 \\
\hline 4 & 18.43 & 361.0921 & 361.0923 & {$[\mathrm{M}+\mathrm{H}]^{+}$} & irigenin & 346.0685 \\
\hline 5 & 21.97 & 387.109 & 387.109 & {$[\mathrm{M}+\mathrm{H}]^{+}$} & irisflorentin & 357.0606 \\
\hline 6 & 10.6 & 493.1342 & 493.134 & {$[\mathrm{M}+\mathrm{H}]^{+}$} & iristectorin $B$ & $331.0815,316.0579$ \\
\hline 7 & 11.1 & 493.1342 & 493.134 & {$[\mathrm{M}+\mathrm{H}]^{+}$} & iristectorin $\mathrm{A}$ & $331.0815,316.0579$ \\
\hline 8 & 11.46 & 523.1447 & 523.1452 & {$[\mathrm{M}+\mathrm{H}]^{+}$} & isoiridin & $523.1447,361.0919$ \\
\hline 9 & 12.78 & 535.1446 & 535.1452 & {$[\mathrm{M}+\mathrm{H}]^{+}$} & 3,5-dimethoxyirisolone-4-O-d-glucoside & $373.0919,358.0685$ \\
\hline 10 & 6.16 & 625.1765 & 625.1767 & {$[\mathrm{M}+\mathrm{H}]^{+}$} & tectorigenin-7-O-glucosyl-4-O-glucoside & $463.1236,301.0707,286.0473$ \\
\hline 11 & 17.98 & 331.0815 & 331.0818 & {$[\mathrm{M}+\mathrm{H}]^{+}$} & iristectorigenin $\mathrm{A}$ & $316.0582,301.0347$ \\
\hline 12 & 18.5 & 331.0813 & 331.0818 & {$[\mathrm{M}+\mathrm{H}]^{+}$} & iristectorigenin B & $316.0582,301.0348$ \\
\hline 13 & 18.12 & 373.0918 & 373.0923 & {$[\mathrm{M}+\mathrm{H}]^{+}$} & noririsflorentin & 358.0687 \\
\hline 14 & 22.37 & 359.0761 & 359.0764 & {$[\mathrm{M}+\mathrm{H}]^{+}$} & dichotomitin & 326.0424 \\
\hline 15 & 17.28 & 357.1342 & 357.1338 & {$[\mathrm{M}-\mathrm{H}]^{-}$} & matairesinol & $342.0380,327.0149$ \\
\hline 16 & 20.02 & 373.1643 & 373.1651 & {$[\mathrm{M}+\mathrm{H}]^{+}$} & arctigenin & 355.1539 \\
\hline 17 & 13.53 & 552.2443 & 552.2445 & {$\left[\mathrm{M}+\mathrm{NH}_{4}\right]^{+}$} & arctiin & $373.1647,355.1542$ \\
\hline 18 & 11.72 & 538.2286 & 538.2288 & {$\left[\mathrm{M}+\mathrm{NH}_{4}\right]^{+}$} & matairesinoside & 359.1491 \\
\hline 19 & 11.99 & 773.2786 & 773.2785 & {$[\mathrm{M}+\mathrm{Na}]^{+}$} & lappaol H & $755.2684,725.2574$ \\
\hline 20 & 18.8 & 559.1938 & 559.1944 & {$[\mathrm{M}+\mathrm{Na}]^{+}$} & lappaol A & 397.1259 \\
\hline 21 & 19.6 & 732.302 & 732.302 & {$\left[\mathrm{M}+\mathrm{NH}_{4}\right]^{+}$} & lappaol F & 531.2014 \\
\hline 22 & 12.8 & 577.2048 & 577.205 & {$[\mathrm{M}+\mathrm{Na}]^{+}$} & lappaol C/isolappaol C/lappaol E/arctignan A & $559.1945,562.1801$ \\
\hline 23 & 13.94 & 577.2048 & 577.205 & {$[\mathrm{M}+\mathrm{Na}]^{+}$} & lappaol C/isolappaol C/lappaol E/arctignan A & $559.1945,562.1804$ \\
\hline 24 & 14.2 & 577.2046 & 577.205 & {$[\mathrm{M}+\mathrm{Na}]^{+}$} & lappaol C/isolappaol C/lappaol E/arctignan A & $559.1947,562.1801$ \\
\hline 25 & 16.1 & 577.2042 & 577.205 & {$[\mathrm{M}+\mathrm{Na}]^{+}$} & lappaol C/isolappaol C/lappaol E/arctignan A & $559.1944,562.1802$ \\
\hline 26 & 9.18 & 417.1187 & 417.1186 & {$[\mathrm{M}-\mathrm{H}]^{-}$} & liquiritin & 255.0653 \\
\hline 27 & 9.34 & 449.1083 & 449.1084 & {$[\mathrm{M}+\mathrm{H}]^{+}$} & galuteolin & 287.0555 \\
\hline 28 & 12.34 & 417.1187 & 417.1186 & {$[\mathrm{M}-\mathrm{H}]^{-}$} & isoliquiritin & 255.0653 \\
\hline 29 & 14.24 & 255.0662 & 255.0657 & {$[\mathrm{M}-\mathrm{H}]^{-}$} & liquiritigenin & 153.0917 \\
\hline 30 & 19.08 & 255.0663 & 255.0657 & {$[\mathrm{M}-\mathrm{H}]^{-}$} & isoliquiritigenin & 153.0918 \\
\hline 31 & 19.8 & 267.0653 & 267.0657 & {$[\mathrm{M}-\mathrm{H}]^{-}$} & formononetin & 252.0431 \\
\hline 32 & 8.86 & 549.1608 & 549.1608 & {$[\mathrm{M}-\mathrm{H}]^{-}$} & liquiritin apioside & $417.1192,255.0665$ \\
\hline 33 & 11.84 & 549.1608 & 549.1608 & {$[\mathrm{M}-\mathrm{H}]^{-}$} & isoliquiritin apioside & $417.1192,255.0666$ \\
\hline 34 & 12.97 & 543.1833 & 543.1866 & {$[\mathrm{M}-\mathrm{H}]^{-}$} & physalin $\mathrm{D}^{\prime}$ & $525.1763,515.1919$ \\
\hline 35 & 15.22 & 543.1833 & 543.1866 & {$[\mathrm{M}-\mathrm{H}]^{-}$} & physalin D & $525.1763,515.1919$ \\
\hline 36 & 17.92 & 525.1759 & 525.1761 & {$[\mathrm{M}-\mathrm{H}]^{-}$} & physalin $\mathrm{F}$ & $507.1663,497.1819$ \\
\hline 37 & 18.18 & 525.176 & 525.1761 & {$[\mathrm{M}-\mathrm{H}]^{-}$} & physalin A & $507.1663,497.1819$ \\
\hline 38 & 18.53 & 527.1915 & 527.1917 & {$[\mathrm{M}-\mathrm{H}]^{-}$} & physalin $\mathrm{O}$ & 509.1815 \\
\hline 39 & 19.01 & 527.1916 & 527.1917 & {$[\mathrm{M}-\mathrm{H}]^{-}$} & physalin L & 509.1815 \\
\hline
\end{tabular}


Table 1. Cont.

\begin{tabular}{|c|c|c|c|c|c|c|}
\hline No. & Retention Time (min) & Experimental Mass $(\mathrm{m} / \mathrm{z})$ & Theoretical Mass $(\mathrm{m} / \mathrm{z})$ & Ion Type & Identification Compound & MS/MS Fragments \\
\hline 40 & 18.22 & 823.4116 & 823.4116 & {$[\mathrm{M}+\mathrm{H}]^{+}$} & glycyrrhizic acid & $647.3791,471.3467,453.3363$ \\
\hline 41 & 30.26 & 471.3464 & 471.3474 & {$[\mathrm{M}+\mathrm{H}]^{+}$} & glycyrrhetinic acid & 425.3416 \\
\hline 42 & 16.89 & 839.407 & 839.4065 & {$[\mathrm{M}+\mathrm{H}]^{+}$} & licorice-saponin G2 & $663.3747,469.3312,451.3210$ \\
\hline 43 & 14.53 & 985.4645 & 985.4644 & {$[\mathrm{M}+\mathrm{H}]^{+}$} & licorice saponin $\mathrm{A} 3$ & $823.4116,647.3806,615.3897,453.3366$ \\
\hline 44 & 6.44 & 423.0923 & 423.0924 & {$[\mathrm{M}+\mathrm{H}]^{+}$} & mangiferin & $405.0819,333.0607,303.0501$ \\
\hline 45 & 6.77 & 423.0923 & 423.0924 & {$[\mathrm{M}+\mathrm{H}]^{+}$} & isomangiferin & $405.0819,333.0607,303.0501$ \\
\hline 46 & 24.63 & 365.1028 & 365.1025 & {$[\mathrm{M}-\mathrm{H}]^{-}$} & glycyrol & $307.0244,295.0244$ \\
\hline 47 & 3.7 & 353.0876 & 353.0873 & {$[\mathrm{M}-\mathrm{H}]^{-}$} & chlorogenic acid & $191.0565,179.0352$ \\
\hline 48 & 4.58 & 353.0876 & 353.0873 & {$[\mathrm{M}-\mathrm{H}]^{-}$} & neochlorogenic acid & $191.0565,179.0352$ \\
\hline 49 & 4.97 & 353.0876 & 353.0873 & {$[\mathrm{M}-\mathrm{H}]^{-}$} & cryptochlorogenic acid & $191.0565,179.0352$ \\
\hline 50 & 8.94 & 515.1188 & 515.119 & {$[\mathrm{M}-\mathrm{H}]^{-}$} & isochlorogenic acid B & 353.0878 \\
\hline 51 & 9.14 & 515.1193 & 515.119 & {$[\mathrm{M}-\mathrm{H}]^{-}$} & isochlorogenic acid A & 353.087 \\
\hline 52 & 9.42 & 515.1191 & 515.119 & {$[\mathrm{M}-\mathrm{H}]^{-}$} & isochlorogenic acid C & 353.0876 \\
\hline 53 & 2.49 & 249.1962 & 249.1967 & {$[\mathrm{M}+\mathrm{H}]^{+}$} & matrine & 148.1122 \\
\hline 54 & 3.14 & 265.1913 & 265.1916 & {$[\mathrm{M}+\mathrm{H}]^{+}$} & oxymatrine & $247.1810,205.1339$ \\
\hline
\end{tabular}


Eleven of the detected ligands (except for peaks 15 and 16) generated adduct ions of $\left[\mathrm{M}+\mathrm{N}_{\mathrm{a}}\right]^{+}$or $\left[\mathrm{M}+\mathrm{NH}_{4}\right]^{+}$in positive mode. By direct comparison with reference compounds, peaks 16 and 17 were readily identified as arctigenin and arctiin, respectively. Based on the accurate masses and literature data, peaks 15, 18, 19, 20, and 21 were identified as matairesinol, matairesinoside, lappaol $\mathrm{H}$, lappaol A, and lappaol F, respectively [12,20]. Peaks 22, 23, 24, and 25 were of isomers with the adduct ions of $[\mathrm{M}+\mathrm{Na}]^{+}$at 577.2048 in positive mode, and they yielded fragment ions at $\left[\mathrm{M}+\mathrm{H}-\mathrm{CH}_{3}\right]^{+}$and $\left[\mathrm{M}+\mathrm{H}-\mathrm{OCH}_{3}\right]^{+}$. These four peaks were ambiguously assigned as lappaol $\mathrm{C}$, isolappaol $\mathrm{C}$, lappaol $\mathrm{E}$, and arctignan $\mathrm{A}$, respectively $[7,12]$.

Six flavonoids were identified by comparisons to reference standards: Liquiritin (peak 26), galuteolin (peak 27), isoliquiritin (peak 28), liquiritigenin (peak 29), isoliquiritigenin (peak 30), and formononetin (peak 31). Peaks 32 and 33 exhibited high-intensity $[\mathrm{M}+\mathrm{H}-132]^{+}$ions and fragment ions formed by the successive loss of $162 \mathrm{Da}$, and were assigned as liquiritin apioside and isoliquiritin apioside, respectively.

Six physalins were detected. Peaks 34 and 35, with the same deprotonated molecular ion at $m / z$ 543.1866 in negative ion mode, represent a pair of stereoisomers. Their parent and product ions were in agreement with physalin $\mathrm{D}$ and physalin $\mathrm{D}^{\prime}$, and the polarity of physalin $\mathrm{D}^{\prime}$ was stronger than that of physalin D; the compound with the shorter retention time should be physalin $\mathrm{D}^{\prime}$ [6]. Using the same method, the other two pairs of stereoisomers, peaks 36 and 37, and peaks 38 and 39, were identified as physalin $\mathrm{F}$ and physalin A, and physalin $\mathrm{O}$ and physalin $\mathrm{L}$, respectively $[6,13,21]$.

The four triterpene saponins were acidic saponins, and glycyrrhizic acid (peak 40) and glycyrrhetinic acid (peak 41) were identified by comparing their retention times and accurate masses with those of the standards. Peak 42 showed $[\mathrm{M}+\mathrm{H}]^{+}$ions at $m / z 839.4070$, which is 16 Da greater than that of glycyrrhizic acid, so it could be assigned to licorice saponin G2 [14]. In positive ion mode, peak 43 gave $[\mathrm{M}+\mathrm{H}]^{+}$at $m / z 985.4645,[\mathrm{M}-162+\mathrm{H}]^{+}$at 823.4116 , and $\left[\mathrm{M}-2 \times 162-\mathrm{H}_{2} \mathrm{O}+\mathrm{H}\right]^{+}$ at $m / z$ 615.3897. Based on the cleavage patterns, which were similar to those of glycyrrhizic acid, the compound was presumed to be licorice saponin A3 [14,22].

Peaks 44 and 45 showed $[\mathrm{M}+\mathrm{H}]^{+}$ions at $m / z 423.0925$ in positive ion mode; this was similar to the accurate masses of mangiferin and isomangiferin $\left([\mathrm{M}+\mathrm{H}]^{+}, 423.0924\right)$. The positive $\mathrm{MS}^{2}$ spectrum of $m / z 423.09$ showed fragment ions at 405.0819, 333.0607, and 303.0501, which correspond to losses of 18,90 , and $120 \mathrm{Da}$, indicating that the fragment ions were $\left[\mathrm{M}+\mathrm{H}-\mathrm{H}_{2} \mathrm{O}\right]^{+},\left[\mathrm{M}+\mathrm{H}-\mathrm{C}_{3} \mathrm{H}_{6} \mathrm{O}_{3}\right]^{+}$, and $\left[\mathrm{M}+\mathrm{H}-\mathrm{C}_{4} \mathrm{H}_{8} \mathrm{O}_{4}\right]^{+}$, resulting from cross-ring cleavages in the sugar moiety. This result is consistent with the cleavage pattern of C-glycosidic xanthone and patterns in the literature [13]. Therefore, peaks 44 and 45 were tentatively identified as mangiferin and isomangiferin.

Peak 46 was tentatively characterized as glycyrol, a licorice coumarin, because of its $[\mathrm{M}-\mathrm{H}]^{-}$ ion at $m / z 365.1028$ and the fragmentation at $m / z 307.0244\left(\left[\mathrm{M}-\mathrm{H}-\mathrm{C}_{4} \mathrm{H}_{10}\right]^{-}\right)$and $m / z 295.0244$ $\left(\left[\mathrm{M}-\mathrm{H}-\mathrm{C}_{5} \mathrm{H}_{10}\right]^{-}\right)$, which were the same as those of glycyrol [23].

Organic acids and alkaloids were identified by comparing their retention times and accurate masses with those of the standards. Peaks 47, 48, and 49 were identified as chlorogenic acid, neochlorogenic acid, and cryptochlorogenic acid, respectively, by comparison with reference compounds. Similarly, because of available standards, peaks 50,51,52, 53, and 54 were unequivocally identified as isochlorogenic acid B, isochlorogenic acid A, sochlorogenic acid C, oxymatrine, and matrine, respectively.

\subsection{Quantitative Analysis}

Extracted ion chromatograms of the standard sample and SJLYKL samples are shown in Figure 2. The results of regression analysis and LOD values for the ten compounds are shown in Table 2. All calibration curves showed good linearity $\left(\mathrm{r}^{2}>0.9914\right)$ between the peak area and concentration. The accuracy of the intra- and inter-day variation of these investigated compounds was 95.0-105.0\% and the precision values were less than 5.0\% (Table 3). The results of the recovery and repeatability test are shown in Table 4 . The recovery of all analytes was 95.1-104.8\%, and the RSD values of 
the repeatability results were less than $4.94 \%$. The sample solution was stable for $24 \mathrm{~h}$ at $4{ }^{\circ} \mathrm{C}$ (RSD $<4.31 \%$, data not shown). Then, the proposed method was applied to analyze ten compounds in five SJLYKL samples. The identified levels of these compounds are summarized in Figure 3. Arctiin was present in the highest concentration, followed by tectoridin. These two components provide good anti-inflammatory effects $[24,25]$ and may be active ingredients in SJLYKL. Therefore, these compounds could be marker compounds for quality control of SJLYKL.
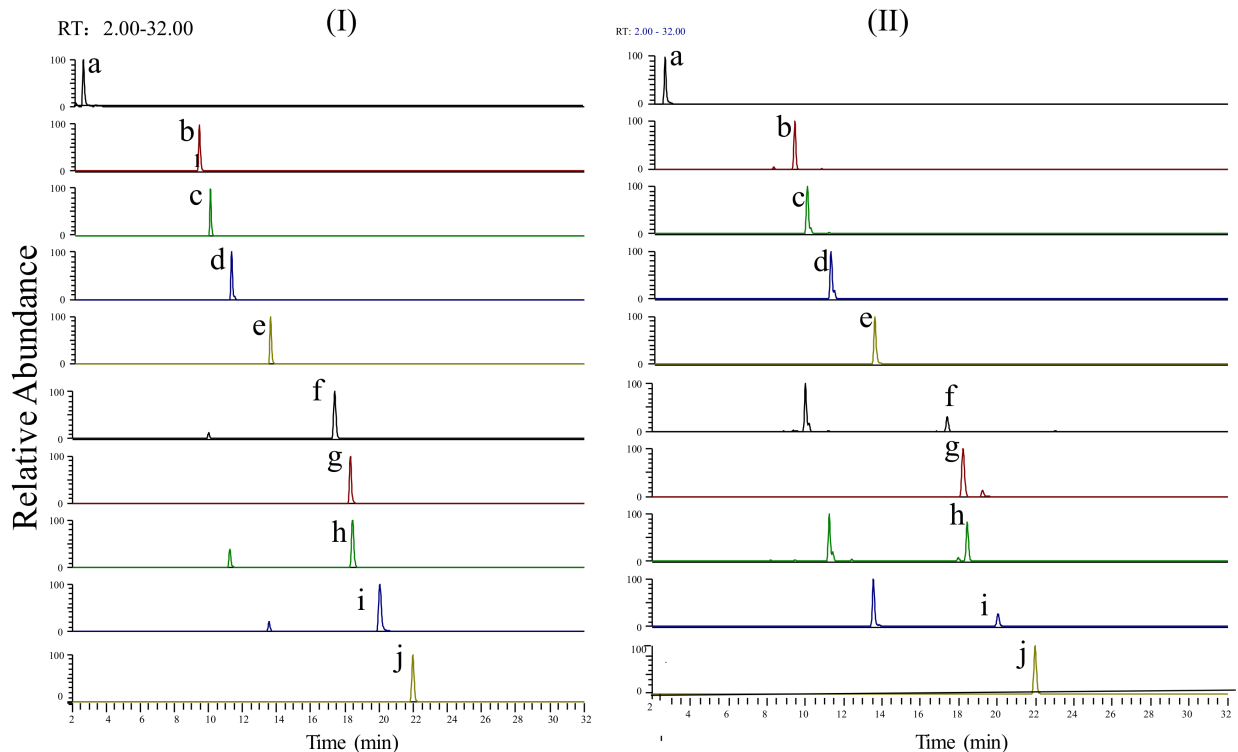

Figure 2. Extracted ion chromatograms of the (I) reference standards and (II) SJLYKL sample: (a) matrine; (b) galuteolin; (c) tectoridin; (d) iridin; (e) arctiin; (f) tectorigenin; (g) glycyrrhizic acid; (h) irigenin; (i) arctigenin; (j) irisflorentin.

Table 2. Regression equation, correlation coefficients, and LOD of the ten compounds.

\begin{tabular}{ccccc}
\hline Compound & Regression Equation & $\mathbf{r}^{\mathbf{2}}$ & Liner Range $(\mu \mathrm{g} / \mathrm{mL})$ & LOD $(\mu \mathrm{g} / \mathrm{mL})$ \\
\hline matrine & $y=46117+5066555 x$ & 0.9914 & $0.020-2.5$ & 0.002 \\
galuteolin & $y=1550+1112359 x$ & 0.9985 & $0.023-3.0$ & 0.002 \\
tectoridin & $y=30165+1743884 x$ & 0.9988 & $0.027-3.5$ & 0.002 \\
iridin & $y=4672+1112803 x$ & 0.9921 & $0.016-2.0$ & 0.002 \\
arctiin & $y=7991+2241923 x$ & 0.9988 & $0.195-25$ & 0.002 \\
tectorigenin & $y=34605+11608484 x$ & 0.9987 & $0.023-3.0$ & 0.002 \\
glycyrrhizic acid & $y=67891+2468972 x$ & 0.9954 & $0.023-3.0$ & 0.002 \\
irigenin & $y=1793+447209 x$ & 0.9972 & $0.025-3.2$ & 0.002 \\
arctigenin & $y=7894+1314941 x$ & 0.9994 & $0.02-2.5$ & 0.002 \\
irisflorentin & $y=54529+50506549 x$ & 0.9989 & $0.016-2.0$ & 0.0005 \\
\hline
\end{tabular}

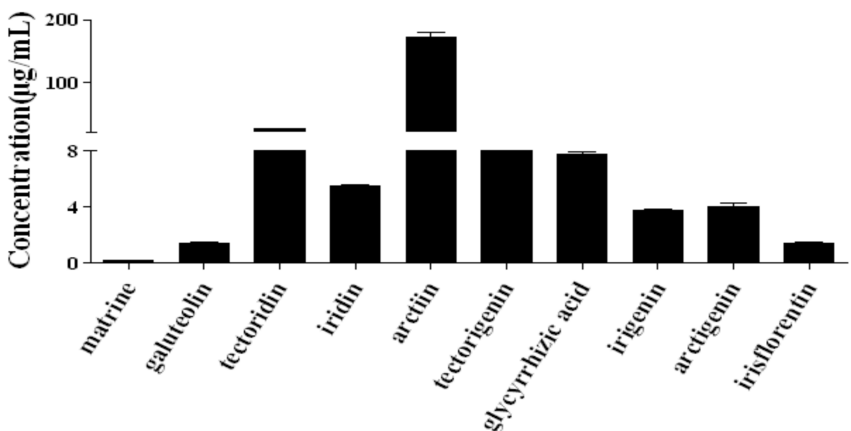

Figure 3. Content determination results of the ten compounds in Shejin-liyan Granule. 
Table 3. Intra- and inter-day precision and accuracy of the ten compounds.

\begin{tabular}{|c|c|c|c|c|c|c|c|c|c|}
\hline \multirow{2}{*}{ Compound } & \multirow{2}{*}{$\begin{array}{l}\text { Concentration } \\
(\mu \mathrm{g} / \mathrm{mL})\end{array}$} & \multicolumn{4}{|c|}{ Intra-Day } & \multicolumn{4}{|c|}{ Inter-Day } \\
\hline & & Mean & \pm & SD & Accuracy (Bias \%) & Mean & \pm & SD & Accuracy (Bias \%) \\
\hline \multirow[t]{3}{*}{ matrine } & 0.040 & 0.038 & \pm & 0.0014 & -5.00 & 0.0398 & \pm & 0.002 & -0.50 \\
\hline & 0.640 & 0.651 & \pm & 0.022 & 1.70 & 0.669 & \pm & 0.015 & 4.50 \\
\hline & 1.875 & 1.782 & \pm & 0.087 & -5.00 & 1.822 & \pm & 0.082 & -2.80 \\
\hline \multirow[t]{3}{*}{ galuteolin } & 0.046 & 0.0438 & \pm & 0.002 & -4.80 & 0.044 & \pm & 0.002 & -4.30 \\
\hline & 0.736 & 0.769 & \pm & 0.028 & 4.50 & 0.755 & \pm & 0.006 & 2.60 \\
\hline & 2.250 & 2.21 & \pm & 0.061 & -1.80 & 2.34 & \pm & 0.059 & 4.00 \\
\hline \multirow[t]{3}{*}{ tectoridin } & 0.054 & 0.052 & \pm & 0.001 & -3.70 & 0.0516 & \pm & 0.001 & -4.40 \\
\hline & 0.864 & 0.811 & \pm & 0.009 & -6.10 & 0.827 & \pm & 0.010 & -4.30 \\
\hline & 2.625 & 2.63 & \pm & 0.102 & 0.20 & 2.56 & \pm & 0.079 & -2.50 \\
\hline \multirow[t]{3}{*}{ iridin } & 0.032 & 0.033 & \pm & 0.001 & 1.90 & 0.0307 & \pm & 0.0004 & -4.10 \\
\hline & 0.512 & 0.531 & \pm & 0.005 & 3.70 & 0.533 & \pm & 0.002 & 4.10 \\
\hline & 1.500 & 1.55 & \pm & 0.069 & 3.30 & 1.543 & \pm & 0.035 & 2.90 \\
\hline \multirow[t]{3}{*}{ arctiin } & 0.400 & 0.42 & \pm & 0.014 & 5.00 & 0.38 & \pm & 0.013 & -5.00 \\
\hline & 6.400 & 6.303 & \pm & 0.046 & -1.50 & 6.21 & \pm & 0.054 & -3.00 \\
\hline & 18.750 & 17.9 & \pm & 0.456 & -4.50 & 17.89 & \pm & 0.432 & -4.60 \\
\hline \multirow[t]{3}{*}{ tectorigenin } & 0.046 & 0.0496 & \pm & 0.0007 & 7.80 & 0.0464 & \pm & 0.0004 & 0.90 \\
\hline & 0.094 & 0.091 & \pm & 0.002 & -2.90 & 0.09 & \pm & 0.003 & -4.00 \\
\hline & 2.250 & 2.15 & \pm & 0.056 & -4.40 & 2.36 & \pm & 0.081 & 4.90 \\
\hline \multirow[t]{3}{*}{ glycyrrhizic acid } & 0.046 & 0.045 & \pm & 0.002 & -2.20 & 0.0438 & \pm & 0.002 & -4.80 \\
\hline & 0.736 & 0.769 & \pm & 0.009 & 4.50 & 0.772 & \pm & 0.009 & 4.90 \\
\hline & 2.250 & 2.32 & \pm & 0.060 & 3.10 & 2.33 & \pm & 0.065 & 3.60 \\
\hline \multirow[t]{3}{*}{ irigenin } & 0.050 & 0.052 & \pm & 0.0008 & 4.00 & 0.0522 & \pm & 0.0004 & 4.40 \\
\hline & 0.800 & 0.797 & \pm & 0.002 & -0.40 & 0.809 & \pm & 0.001 & 1.10 \\
\hline & 2.400 & 2.32 & \pm & 0.016 & -3.30 & 2.35 & \pm & 0.018 & -2.10 \\
\hline \multirow[t]{3}{*}{ arctigenin } & 0.040 & 0.038 & \pm & 0.001 & -5.00 & 0.0391 & \pm & 0.001 & -2.20 \\
\hline & 0.640 & 0.653 & \pm & 0.005 & 2.00 & 0.652 & \pm & 0.006 & 1.90 \\
\hline & 1.875 & 1.86 & \pm & 0.053 & -0.80 & 1.85 & \pm & 0.071 & -1.30 \\
\hline \multirow[t]{3}{*}{ irisflorentin } & 0.032 & 0.0336 & \pm & 0.0003 & 5.00 & 0.031 & \pm & 0.0004 & -3.10 \\
\hline & 0.500 & 0.512 & \pm & 0.001 & 2.40 & 0.513 & \pm & 0.001 & 2.60 \\
\hline & 1.500 & 1.465 & \pm & 0.013 & -2.30 & 1.457 & \pm & 0.015 & -2.90 \\
\hline
\end{tabular}

Table 4. Recovery and repeatability of these ten compounds $(n=3)$.

\begin{tabular}{cccccccccc}
\hline \multirow{2}{*}{ Compound. } & \multicolumn{4}{c}{ Recovery } & \multicolumn{4}{c}{ Reproducibility $(\mu \mathrm{g} / \mathrm{mL})$} \\
\cline { 2 - 9 } & Spiked Amount $(\mu \mathrm{g})$ & \multicolumn{1}{c}{ Dectected Amount $(\mu \mathrm{g})$} & Accuracy (Bias \%) & Mean & \pm & SD & RSD \\
\hline matrine & 1.0 & 1.027 & \pm & 0.04 & 4.80 & 0.19 & \pm & 0.003 & 1.67 \\
galuteolin & 7.3 & 7.28 & \pm & 0.25 & -0.10 & 1.39 & \pm & 0.03 & 2.27 \\
tectoridin & 125.8 & 119.6 & \pm & 5.85 & -4.90 & 24.33 & \pm & 0.53 & 2.18 \\
iridin & 27.3 & 26.9 & \pm & 1.23 & -1.50 & 5.68 & \pm & 0.25 & 4.40 \\
arctiin & 863.5 & 862.1 & \pm & 31.56 & -0.20 & 169.60 & \pm & 2.50 & 1.47 \\
tectorigenin & 56.7 & 55.6 & \pm & 1.52 & -1.90 & 11.00 & \pm & 0.54 & 4.91 \\
glycyrrhizic acid & 38.8 & 37.1 & \pm & 0.64 & -4.50 & 7.49 & \pm & 0.37 & 4.94 \\
irigenin & 19.0 & 18.2 & \pm & 0.19 & -4.30 & 3.92 & \pm & 0.15 & 3.83 \\
arctigenin & 20.3 & 19.5 & \pm & 0.75 & -3.90 & 4.25 & \pm & 0.19 & 4.47 \\
irisflorentin & 7.2 & 6.9 & \pm & 0.26 & -3.80 & 1.37 & \pm & 0.07 & 4.91 \\
\hline
\end{tabular}

\section{Conclusions}

In this study, a simple, accurate, and reliable HPLC-LTQ-Orbitrap MS method was established to qualitatively determine the 54 components of SJLYKL. The method successfully quantified ten major components in five batches of SJLYKL samples. This novel approach was useful to identify constituents and control the quality of SJLYKL. These results offer useful information for understanding the material basis of the therapeutic effects of SJLYKL and for its clinical application.

Supplementary Materials: The chemical structures of the 54 compounds are available online.

Author Contributions: Jifeng Gu, Xinhua Liu, and Yizhun Zhu participated in research design. Jifeng Gu, Weijun $\mathrm{Wu}$, and Mengwei Huang conducted experiments. Jifeng $\mathrm{Gu}$ and Fen Long performed data analysis. Jifeng Gu, Xinhua Liu, and Yizhun Zhu wrote the manuscript.

Conflicts of Interest: The authors declare no conflict of interest. 


\section{References}

1. Uemura, J.; Nagpal, R.; Zerbinati, N.; Singh, B.; Marcellino, M.; Mohania, D.; Mohania, D.; Marotta, F.; He, F.; Ayala, A.; et al. Effect of VBC-1814/7J, a poly-phytocompound, on a non-infectious model of pharyngitis. Exp. Ther. Med. 2017, 13, 3075-3080. [CrossRef] [PubMed]

2. Spinks, A.; Glasziou, P.P.; Del Mar, C.B. Antibiotics for sore throat. Cochrane Database Syst. Rev. 2013, 11, CD000023. [CrossRef] [PubMed]

3. Huang, Y.; Wu, T.; Zeng, L.; Li, S. Chinese medicinal herbs for sore throat. Cochrane Database Syst. Rev. 2012, 3, CD004877. [CrossRef] [PubMed]

4. Pelucchi, C.; Grigoryan, L.; Galeone, C.; Esposito, S.; Huovinen, P.; Little, P.; Verheij, T. Guideline for the management of acute sore throat. Clin. Mocrobiol. Infect. 2012, 18, 1-27. [CrossRef] [PubMed]

5. Li, J.; Li, W.Z.M.; Huang, W.; Cheung, A.W.H.; Bi, C.W.C.; Duan, R.; Guo, A.J.Y.; Dong, T.T.X.; Tsim, K.W.K. Quality evaluation of Rhizoma Belamcandae (Belamcanda chinensis (L.) DC.) by using high-performance liquid chromatography coupled with diode array detector and mass spectrometry. J. Chormatogr. A 2009, 1216, 2071-2078. [CrossRef] [PubMed]

6. Feng, X.; Huo, X.; Liu, H.; Chai, L.; Ding, L.; Qiu, F. Identification of absorbed constituents and in vivo metabolites in rats after oral administration of Physalis alkekengi var. franchetii by ultra-high-pressure liquid chromatography quadrupole time-of-flight mass spectrometry. Biomed. Chromatogr. 2017, 32, e4121.

7. Liu, J.; Cai, Y.-Z.; Wong, R.N.S.; Lee, C.K.-F.; Tang, S.C.W.; Sze, S.C.W.; Tong, Y.; Zhang, Y. Comparative Analysis of Caffeoylquinic Acids and Lignans in Roots and Seeds among Various Burdock (Arctium lappa) Genotypes with High Antioxidant Activity. J. Agric. Food Chem. 2012, 60, 4067-4075. [CrossRef] [PubMed]

8. Tang, L.; Dong, L.-N.; Peng, X.-J.; Li, Y.; Shi, J.; Zhou, F.-Y.; Liu, Z.Q. Pharmacokinetic characterization of oxymatrine and matrine in rats after oral administration of radix Sophorae tonkinensis extract and oxymatrine by sensitive and robust UPLC-MS/MS method. J. Pharm. Biomed. 2013, 83, 179-185. [CrossRef] [PubMed]

9. Kang, H.K.S.; Choi, H.Y. Inhibitory effect of Physalis alkekengi L. var. franchetii extract and its chloroform fraction on LPS or LPS/IFN- $\gamma$-stimulated inflammatory response in peritoneal macrophages. J. Ethnopharmacol. 2011, 135, 95-101. [CrossRef] [PubMed]

10. Orhan, I.N.S.; Sener, B.; Ayanoglu, F.; Ozgüven, M.; Choudhary, M.I.; Ur-Rahman, A. Two isoflavones and bioactivity spectrum of the crude extracts of Iris germanica rhizomes. Phytother. Res. 2003, 17, 575-577. [CrossRef] [PubMed]

11. Park, S.Y.; Hong, S.S.; Han, X.H.; Hwang, J.S.; Lee, D.; Ro, J.S.; Hwang, B.Y. Lignans from Arctium lappa and their inhibition of LPS-induced nitric oxide production. Chem. Pharm. Bull. 2007, 55, 150-152. [CrossRef] [PubMed]

12. Hu, J.Q.K.; Tingting, Z.; Xiaoli, W.; Mengxue, M.; Baochang, C. Qualitative and quantitative analysis of major constituents of raw and processed Arctii Fructus by UHPLC-UV-Q-TOF-MS/MS. Acta Pharm. Sin. 2017, 52, 603-608.

13. Zhang, Y.-Y.; Wang, Q.; Qi, L.-W.; Qin, X.-Y.; Qin, M.-J. Characterization and determination of the major constituents in Belamcandae Rhizoma by HPLC-DAD-ESI-MSn. J. Pharm. Biomed. 2011, 56, 304-314. [CrossRef] [PubMed]

14. Qi, Y.; Li, S.; Pi, Z.; Song, F.; Lin, N.; Liu, S.; Liu, Z. Chemical profiling of Wu-tou decoction by UPLC-Q-TOF-MS. Talanta 2014, 118, 21-29. [CrossRef] [PubMed]

15. Cheng, Q.; Shou, L.; Chen, C.; Shi, S.; Zhou, M. Application of ultra-high-performance liquid chromatography coupled with LTQ-Orbitrap mass spectrometry for identification, confirmation and quantitation of illegal adulterated weight-loss drugs in plant dietary supplements. J. Chormatogr. B 2017, 1064, 92-99. [CrossRef] [PubMed]

16. Dias, A.L.S.; Rozet, E.; Larondelle, Y.; Hubert, P.; Rogez, H.; Quetin-Leclercq, J. Development and validation of an UHPLC-LTQ-Orbitrap MS method for non-anthocyanin flavonoids quantification in Euterpe oleracea juice. Anal. Bioanal. Chem. 2013, 405, 9235-9249. [CrossRef] [PubMed]

17. Farré, M.; Picó, Y.; Barceló, D. Application of ultra-high pressure liquid chromatography linear ion-trap orbitrap to qualitative and quantitative assessment of pesticide residues. J. Chormatogr. A 2014, 1328, 66-79. [CrossRef] [PubMed]

18. Woźniak, D.M.A. Belamcandae chinensis rhizome-A review of phytochemistry and bioactivity. Fitoterapia 2015, 107, 1-14. [CrossRef] [PubMed] 
19. Zhang, L.; Wei, K.; Xu, J.; Yang, D.; Zhang, C.; Wang, Z.; Li, M. Belamcanda chinensis (L.) DC-An ethnopharmacological, phytochemical and pharmacological review. J. Ethnopharmacol. 2016, 186, 1-13. [PubMed]

20. He, L.; Chen, Y.; Liang, Z.; Li, Y.; Zhou, M.; Yuan, Z.; Luo, L.; Jin, Z.; Yang, Y.; Chen, J. A rapid and comprehensive quality assessing method of Yin-Qiao-Jie-Du tablets using UHPLC-QTOF-MS in combination with multivariate statistical analysis. J. Pharm. Biomed. 2016, 124, 129-137. [CrossRef] [PubMed]

21. Huang, C.; Xu, Q.; Chen, C.; Song, C.; Xu, Y.; Xiang, Y.; Feng, Y.; Ouyang, H.; Zhang, Y.; Jiang, H. The rapid discovery and identification of physalins in the calyx of Physalis alkekengi L.var.franchetii (Mast.) Makino using ultra-high performance liquid chromatography-quadrupole time of flight tandem mass spectrometry together with a novel three-step data mining strategy. J. Chormatogr. A 2014, 1361, 139-152.

22. Dong, H.; Yan, G.-L.; Han, Y.; Sun, H.; Zhang, A.-H.; Li, X.-N.; Wang, X.-J. UPLC-Q-TOF/MS-based metabolomic studies on the toxicity mechanisms of traditional Chinese medicine Chuanwu and the detoxification mechanisms of Gancao, Baishao, and Ganjiang. Chin. J. Nat. Med. 2015, 13, 687-698. [CrossRef]

23. Wang, Q.; Qiao, X.; Qian, Y.; Liu, C.-F.; Yang, Y.-F.; Ji, S.; Li, J.; Guo, D.-A.; Ye, M. Metabolites identification of glycyrin and glycyrol, bioactive coumarins from licorice. J. Chormatogr. B 2015, 983-984, 39-46. [CrossRef] [PubMed]

24. Lee, S.; Shin, S.; Kim, H.; Han, S.; Kim, K.; Kwon, J.; Kwak, J.-H.; Lee, C.-K.; Ha, N.-J.; Yim, D. Anti-inflammatory function of arctiin by inhibiting COX-2 expression via NF- $\mathrm{B}$ pathways. J. Inflamm. 2011, 8, 16. [CrossRef] [PubMed]

25. Kim, Y.P.Y.M.; Lim, S.S.; Lee, S.H.; Ryu, N.; Shin, K.H.; Ohuchi, K. Inhibition by tectorigenin and tectoridin of prostaglandin E2 production and cyclooxygenase-2 induction in rat peritoneal macrophages. Biochim. Biophys. Acta 1999, 1438, 399-407. [CrossRef]

(C) 2018 by the authors. Licensee MDPI, Basel, Switzerland. This article is an open access article distributed under the terms and conditions of the Creative Commons Attribution (CC BY) license (http://creativecommons.org/licenses/by/4.0/). 